\title{
Microscopic origins of the surface exciton photoluminescence in $\mathrm{ZnO}$ nanostructures
}

\author{
Mahua Biswas ${ }^{a^{*}}$, Yun Suk Jung ${ }^{b}$, Hong Koo Kim ${ }^{b}$, Kumarappan Kumar ${ }^{c}$, Gregory J. Hughes ${ }^{c}$, S. \\ Newcomb ${ }^{d}$, Martin O. Henry ${ }^{a}$, Enda McGlynn ${ }^{a}$ \\ ${ }^{a}$ School of Physical Sciences, National Centre for Plasma Science and Technology, Dublin City \\ University, Glasnevin, Dublin 9, Ireland \\ ${ }^{b}$ Department of Electrical and Computer Engineering, Petersen Institute of NanoScience and \\ Engineering, University of Pittsburgh, Pittsburgh, PA 15260, USA \\ ${ }^{c}$ School of Physical Sciences, National Centre for Sensor Research, Dublin City University, \\ Glasnevin, Dublin 9, Ireland \\ ${ }^{d}$ Glebe Scientific Ltd., Newport, Co. Tipperary, Ireland
}

*Author to whom correspondence should be addressed: mahua.biswas2@mail.dcu.ie.

\begin{abstract}
Photoluminescence (PL) studies of the surface exciton peak in $\mathrm{ZnO}$ nanostructures at $\sim 3.367 \mathrm{eV}$ are reported to elucidate the nature and origin of the emission and its relationship to nanostructure morphology. Localised voltage application in high vacuum and different gas atmospheres show a consistent PL variation (and recovery), allowing an association of the PL to a bound excitonic transition at the $\mathrm{ZnO}$ surface modified by an adsorbate. Studies of samples treated by plasma and of samples exposed to UV light under high vacuum conditions show no consistent effects on the surface exciton peak indicating no involvement of oxygen species. X-ray photoelectron spectroscopy data indicate involvement of adsorbed $\mathrm{OH}$ species. The relationship of the surface exciton peak to the nanostructure morphology is discussed in light of x-ray diffraction, scanning and transmission electron microscopy data.
\end{abstract}

Keyword: Nanostructure, photoluminescence, surface exciton, adsorption

\section{Introduction}

$\mathrm{ZnO}$ nanostructures provide an ideal system to study the influence of surface effects on optical properties due to their large surface-to-volume ratios. Photoluminescence (PL) studies of bulk and nanostructured $\mathrm{ZnO}$ material reveal a range of near bandedge excitonic emission lines, mostly donor bound exciton (DBE) lines, at low temperature, denoted by the labels $\mathrm{I}_{0}$ to $\mathrm{I}_{11}{ }^{1}$ which are visible in both bulk and nanostructured $\mathrm{ZnO}$ samples. However, an asymmetric peak, broader than the I lines $(\sim 5 \mathrm{meV})$, at $\sim 3.367 \mathrm{eV}$, denoted as $\mathrm{I}_{2}$ by Meyer et al., ${ }^{1}$ and as a surface-related bound exciton (SX) 
peak by various authors, can be seen also, mostly in nanostructured materials. ${ }^{2-7}$. This was observed in freshly cleaved $\mathrm{ZnO}$ crystals by Travnikov et al. ${ }^{8}$ and more recently only in emission from $\mathrm{ZnO}$ nanostructures with high surface-to-volume ratios. $2,3,5,6,9,10$ These properties, and the parallels with similar emissions observed in a range of other materials, ${ }^{4,11-14}$ have led to its labelling as a bound exciton transition at a surface or near surface defect. Although the SX peak itself is visible only at temperatures below $25 \mathrm{~K}$, nanostructures showing the SX peak also have rapidly decaying bandedge intensity with increasing temperature ${ }^{7}$ and it appears that the surface conditions responsible for the SX peak lead also to temperature activated non-radiative recombination processes at higher temperatures.

There are many reports on SX peak emission in $\mathrm{CdS}$ and $\mathrm{ZnO}$ materials which provide evidence for the surface nature of the SX peak emission 2, 5, 6, 9-13, 15, 21 but a number of gaps in understanding remain including whether the $\operatorname{defect}(\mathrm{s})$ responsible are crystal defects confined to the (sub-) surface region $^{17}$ or adsorbed surface species which can bind excitons in their vicinity. Adsorption and/or chemisorption processes at semiconductor surfaces, and specifically in the case of ZnO. ${ }^{18,19,20}$ Adsorbates such as $\mathrm{O}_{2}^{-}, \mathrm{O}^{-}, \mathrm{O}^{2-}, \mathrm{OH}^{-}, \mathrm{H}_{2} \mathrm{O}$ etc. have been considered previously as possible origins of the SX band, 5, 6, 9,21 with particular attention on $\mathrm{O}$ and $\mathrm{O}_{2}$, but no conclusion has been reached. Also, the assignment of the large surface-to-volume ratio of nanostructures as the sole or main determinant of the SX peak relative intensity is questionable given the existence of a number of reports of low temperature $\mathrm{PL}$ from $\mathrm{ZnO}$ nanostructures with varying aspect ratios, which show no consistent correlation from one report to another between the SX peak relative intensity and the nanostructure aspect ratio (as well as variations with morphology variations seen within individual reports) see e.g. ref 15, 22-24.

In this work, we study the effect on the SX peak intensity of localised voltage application in high vacuum and different gas atmospheres. We also study the effects on the SX peak of desorbing surface oxygen species using different surface treatments including well-established methods such as plasma treatment and UV exposure under high vacuum conditions. X-ray photoelectron spectroscopy (XPS) studies of UV illuminated samples have been made in the O1s spectrum region before and after UV illumination to study the surface adsorbed species. The effects of nanostructure morphology and crystallinity have been studied using X-ray diffraction (XRD), scanning and transmission electron microscopy (SEM and TEM, respectively) studies. Based on all these data we conclude that the origin of the peak is due to a transition at an exciton bound at $\mathrm{ZnO}$ surfaces modified by adsorbed $\mathrm{OH}$ and that the detailed morphology of the $\mathrm{ZnO}$ nanostructures is important in determining the intensity of the SX signal.

\section{Experimental}

The $\mathrm{ZnO}$ nanostructure growth technique is described in detail elsewhere. ${ }^{25,}{ }^{26}$ Briefly, $\mathrm{ZnO}$ nanostructures were grown on Au-catalysed $a$-plane (11-20) sapphire substrates and Si substrates using vapour phase transport (VPT). A $5 \mathrm{~nm}$ Au layer was evaporated onto $5 \mathrm{~mm}$ diameter circular or $9 \mathrm{~mm}^{2}$ square continuous areas on the ultrasonically cleaned substrates using a thermal evaporator. The furnace temperature is set at either $900^{\circ} \mathrm{C}$ or $950^{\circ} \mathrm{C}$ and samples were grown for 60 minutes. 
PL spectra have been acquired using a Bomem DA8 FT spectrometer with the samples in a closed cycle cryostat (Janis Research). All PL spectra in this study were taken with the same instrumental setup. The detector aperture enabled a spectral resolution of $5 \mathrm{~cm}^{-1}(\sim 0.4 \mathrm{meV})$. In all spectra the SX peak has been compared to the other two main peaks $\left(I_{6}\right.$ and $\left.I_{9}\right)$ in terms of peak intensity (not integrated intensity) since spectral linewidths were largely unchanged for all features.

Electrical voltages in vacuum, air and He gas atmospheres have been applied in-situ in the cryostat sample chamber (to avoid, insofar as possible, adsorption of species from ambient during transfer from one chamber to another). Samples used for this study were grown on $\mathrm{Si}$ and an insulating 500 $\mathrm{nm} \mathrm{SiO}_{2}$ layer was deposited on the $\mathrm{Si}$ using plasma enhanced chemical vapour deposition (PECVD), before $\mathrm{ZnO}$ deposition. Voltages were applied for 1 hour and the leakage current level varied in the range $10-20 \mathrm{~mA}$. Both $n$-type (resistivity $2.5-8 \mathrm{ohm} \mathrm{cm}$ ) and p-type (resistivity 5-9 ohm $\mathrm{cm}) \mathrm{Si}$ were used and both positive and negative voltages were applied to the nanostructures ranging from $40 \mathrm{~V}$ to $70 \mathrm{~V}$. Changes in the SX peak intensity were seen only from $50 \mathrm{~V}$ upwards. At $70 \mathrm{~V}$ and above the samples were damaged due to breakdown of the $\mathrm{SiO}_{2}$ layer. Following voltage application, low temperature PL data were taken from regions adjacent (at a distance of $\sim 2 \mathrm{~mm}$ ) to the electrical contact region in the $\mathrm{ZnO}$ nanostructure deposition area, with the sample always cooled in a $\mathrm{He}$ atmosphere.

The effects of a variety of surface treatments on the SX line intensity were studied. Plasma treatments were undertaken using both $\mathrm{O}$ and Ar plasmas. The $\mathrm{O}$ plasma was produced with an inductively coupled plasma reactive ion etching (ICP-RIE) system (Unaxis 790 ICP-RIE) with an ICP power of $125 \mathrm{~W}$ and an RIE power of $5 \mathrm{~W}$, O pressure of $15 \mathrm{mTorr}$, and a flow rate of $30 \mathrm{sccm}$ for 30 minutes. The Ar plasma was produced using the same instrument and conditions except the ICP power and time were reduced to $50 \mathrm{~W}$ and 5 minutes, respectively. In both plasmas, the RIE power was maintained at the minimum level in order to alleviate possible plasma damage to the $\mathrm{ZnO}$ samples. The $\mathrm{ZnO}$ nanostructures were exposed to the plasma without intentional heating of the sample.

UV illumination experiments were usually performed on the sample in the cryostat (similar to electrical voltage experiments) using a $250 \mathrm{~W}$ iron doped metal halide UV lamp (UV-H $253 \mathrm{BL}-$ UV Light Technology Ltd.). Samples were also illuminated in the XPS chamber described later. The spectral output of the lamp is from $280 \mathrm{~nm}$ to $450 \mathrm{~nm}(4.42 \mathrm{eV}$ to $2.75 \mathrm{eV})$. PL spectra were taken before illumination and the temperature was then raised to room temperature and the sample chamber of the cryostat evacuated to $\sim 10^{-6}$ mbar. The samples were illuminated for a range of times (6 hours, 8 hours, 20 hours and 24 hours) and power densities of $\sim 22 \mathrm{~mW} / \mathrm{cm}^{2}, 15 \mathrm{~mW} / \mathrm{cm}^{2}$ and 3 $\mathrm{mW} / \mathrm{cm}^{2}$, were obtained with a power meter at distances, respectively, of $0 \mathrm{~cm}$ (i.e. lamp directly touching meter), $3.5 \mathrm{~cm}$ (the sample distance within PL chamber) and $18 \mathrm{~cm}$ (the sample distance within the XPS chamber) from the lamp. The samples were then cooled to low temperatures to judge the effects on the SX PL peak.

Material surface composition before and after UV illumination were studied using XPS at base pressures in the preparation and analysis chambers of $2 \times 10^{-6}$ and $1 \times 10^{-9}$ mbar, respectively, using an $\mathrm{Al} \mathrm{K} \alpha(\mathrm{hv}=1486.6 \mathrm{eV}) \mathrm{x}$-ray source. The pass energy of the analyser was set at $20 \mathrm{eV}$ yielding a resolution of approximately $1.0 \mathrm{eV}$. Binding energy scale calibration was performed with the $\mathrm{C} 1 \mathrm{~s}$ 
line $(285 \mathrm{eV})$ from the adventitious carbon contamination layer. ${ }^{27}$ The XPS peaks were fitted with mixed ratio of Gaussian and Lorentzian line shapes and a Shirley background function.

Scanning electron microscopy (SEM) images were taken using a field emission SEM (FESEM) system (Hitachi S-4300 field emission system) and a $\mathrm{LaB}_{6}$ emitter system (Karl-Zeiss EVO series). XRD was performed using a Bruker AXS D8 advance texture diffractometer. TEM studies were performed using a JEOL2000FX system operating at $200 \mathrm{kV}$.

\section{Results}

\subsection{SX peak behavior after electrical voltage application}

In figure 1 (a)-(c) PL spectra for samples where voltages of $60 \mathrm{~V}$ were applied in vacuum are shown. For voltages of $50 \mathrm{~V}$ the same changes as shown in figure 1 (a)-(c) have been observed (data not shown). From figure 1 (b) it is observed that the SX peak relative intensity has increased significantly compared to the I-line peaks after voltage application. The notable point is that the SX peak relative intensity dropped down again to its initial value after exposure to air, as shown in figure 1 (c).

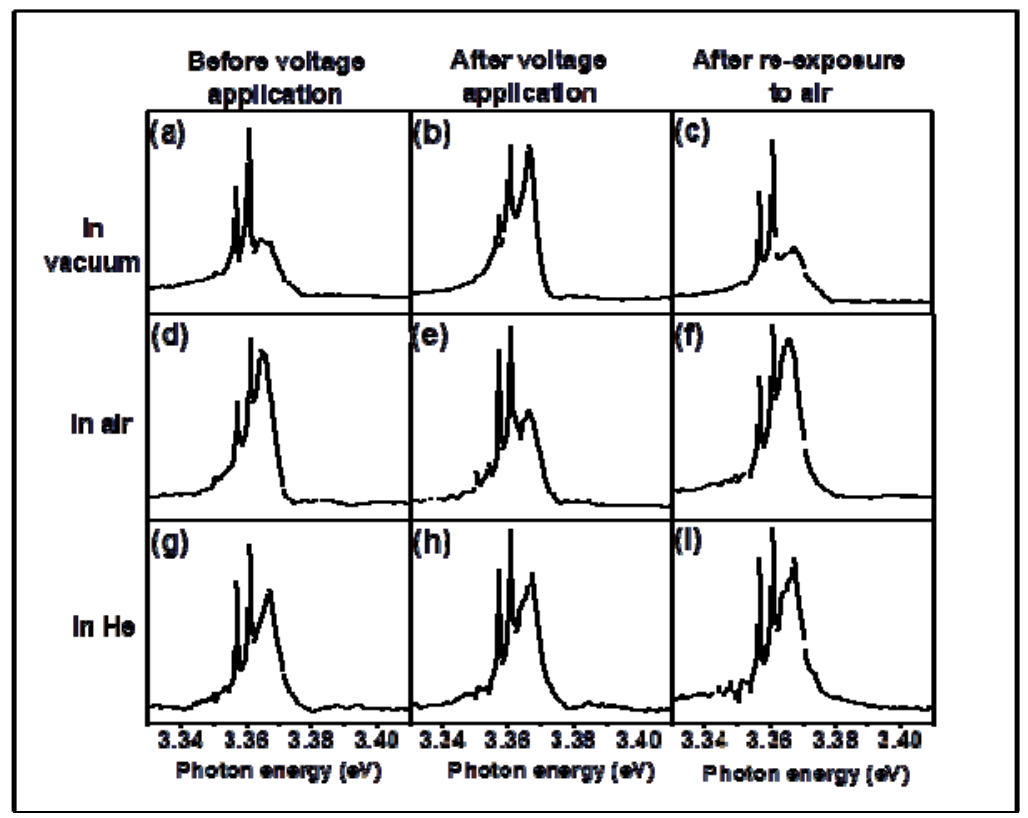

Figure 1: PL study by electrical voltage (60V) application in vacuum (a)-(c), air (d)-(f) and He gas (g)-(i). (a), (d) and (g) are PL spectra taken at $18 \mathrm{~K}$ before electric voltage application, (b), (e) and (h) are PL spectra taken at $18 \mathrm{~K}$ after electric voltage application and (c), (f) and (i) are PL spectra taken at $18 \mathrm{~K}$ after re-exposure to air without voltage, respectively (PL intensity scales are linear).

PL spectra for samples where voltages of $60 \mathrm{~V}$ were applied in air and He are shown in figure 1 (d)(f) and (g)-(i), respectively. All of the experiments in air and He used a fixed positive voltage of $60 \mathrm{~V}$. After voltage application in air the relative intensity of the SX peak has decreased (figure 1(e)) and after re-exposure to ambient the SX peak increases again and returns to its initial level as shown 
in figure 1 (f). After voltage application in He, shown in figure $1(\mathrm{~h})$, the relative intensity of the SX peak remains identical to that before voltage application shown in figure $1(\mathrm{~g})$, and after re-exposure to ambient it remains unchanged (figure 1 (i)). We note that in all cases following voltage application the changes in the PL spectra remain after the voltage is turned off and the sample electrical connections grounded. The spectra only recover to their pre-voltage application levels when actually re-exposed to air over timescales of at least 12 hours. These data were consistently reproducible on a large number of samples. Also, no differences in SX relative intensity were seen for experiments with positive applied biases compared to negative in vacuum and for experiments with $p$-type Si substrate compared to $n$-type $\mathrm{Si}$ substrate (data not shown).

\subsection{SX peak behaviour after plasma treatment}

Plasma treatments with both $\mathrm{O}$ and Ar plasmas were performed on $\mathrm{ZnO}$ nanostructure samples grown on $a$-sapphire at $900^{\circ} \mathrm{C}$. From the FESEM image in figure 2 (a) of a sample treated with the $\mathrm{O}$ plasma we can see the nanorods were physically damaged during the plasma treatment and almost all the nanorods and some nanowalls were displaced from the substrate. However the PL spectra in figures 2 (c) and (d) show no significant change in the SX peak compared to the other I line peaks.

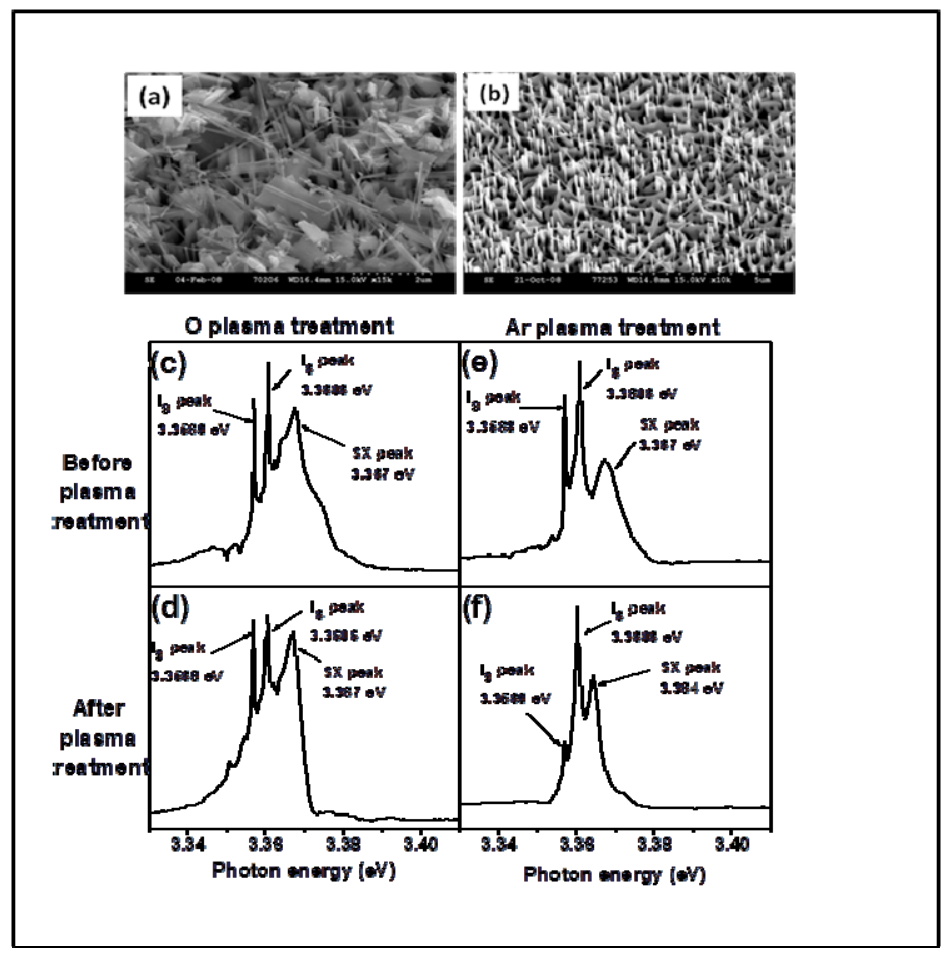

Figure 2: (a) and (b) FESEM images of $\mathrm{O}$ and Ar plasma treated sample, respectively, after plasma treatment, (c) and (d) PL spectra at $18 \mathrm{~K}$ of the sample shown in (a) before and after $\mathrm{O}$ plasma treatment, respectively. (e) and (f) are the PL spectra of the sample at $18 \mathrm{~K}$ shown in (b) before and after Ar plasma treatment, respectively (PL intensity scales are linear). 
An Ar plasma treatment was undertaken next with reduced ICP power and treatment time to reduce nanorod damage. From the FESEM images of sample in figure 2 (b) we observe that in this experiment the damage to the nanorods is much less than that seen in the O plasma treatment, but nevertheless some nanorods are still physically displaced. The PL spectrum after plasma treatment in figure 2 (f) shows no significant decrease in the SX peak intensity compared to the as grown sample spectra in figure 2 (e). There are some slight changes in the spectral shape in both $\mathrm{O}$ and Ar plasma treated samples, especially at the higher photon energies where the surface treatment may have affected the free-exciton polariton properties and escape probability, but the overall relative intensity at the SX peak region has hardly changed and in fact it has increased slightly.

\subsection{SX peak behavior after UV illumination}

PL spectra of four sets of experiments are shown below in figure 3 comprising sets of data from four different samples grown on $a$-sapphire at $900^{\circ} \mathrm{C}$. The broad peak at $\sim 3.367 \mathrm{eV}$ in all spectra in figure 3 is the SX peak. For the 6 hours illumination period we can see the relative intensity of the SX peak is almost the same (figure 3 (b)), while for 8 hours and 20 hours illumination this peak intensity increases (figure 3 (e) and (h), respectively) after UV light illumination. For 24 hour illumination the SX peak intensity is almost the same in all spectra, but the linewidth has narrowed slightly after UV illumination (figure $3(\mathrm{k})$ ).

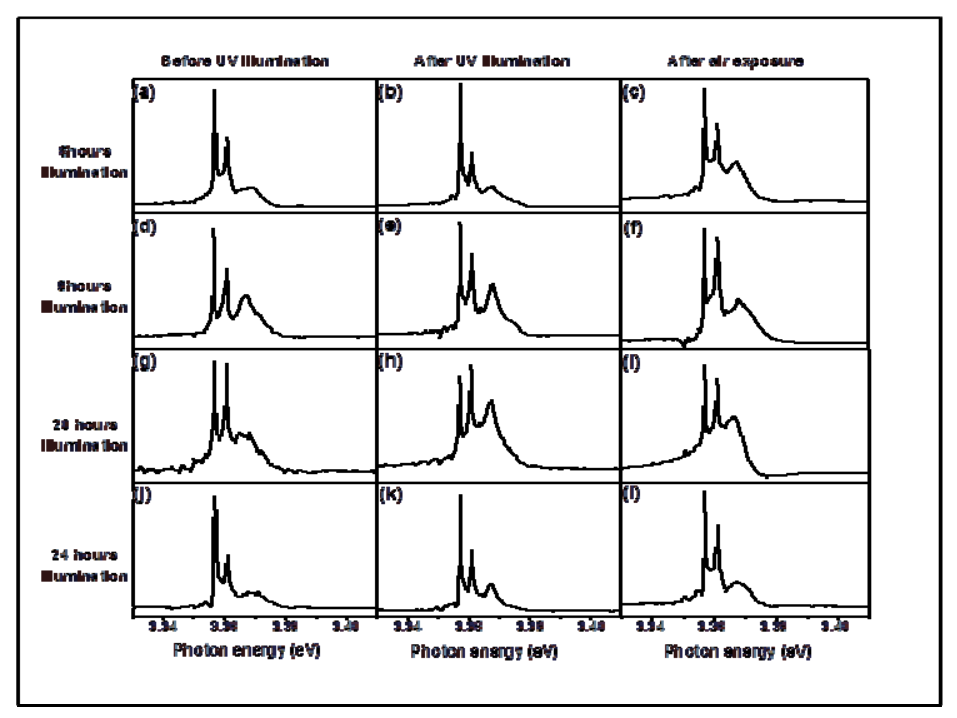

Figure 3: PL spectra (at $18 \mathrm{~K}$ ) of $\mathrm{ZnO}$ nanostructure samples illuminated with UV light for different time durations. Illumination time; (a)-(c) 6hours, (d)-(f) 8 hours, (g)-(i) 20 hours, (j)-(l) 24 hours (PL intensity scales are linear). The first column indicates the spectra before illumination, the second column after illumination, and the third column after re-exposure to air (post UV illumination).

After UV illumination and measurement these samples were taken out to air (for a minimum of 12 hours in all cases), and the PL spectra were then measured again (in few experiments samples were taken out to $\mathrm{O}_{2}$ also). There is no evidence of significant and consistant changes in the SX peak after exposure to air for all the samples as shown in figure 3 (c), (f), (i) and (1). 


\subsection{XPS study of UV illuminated samples}

XPS studies of ZnO nanostructure samples showing intense SX peaks in PL were undertaken and specifically the XPS spectra were taken before UV illumination and after UV illumination in ultrahigh vacuum $\left(\sim 10^{-9}\right.$ mbar) within the XPS chamber to investigate any surface compositional changes. It has always been observed in XPS studies of various $\mathrm{ZnO}$ samples that the $\mathrm{O}$ peak is more sensitive to compositional changes compared to the $\mathrm{Zn}$ peak, ${ }^{28}$ hence we concentrate on the $\mathrm{O}$ region of the spectrum. The detailed peak fittings of the O1s spectra is shown in figure 4 and it indicates that the peak profile is consistent with the observation of four component peaks, two of which are $\mathrm{ZnO}$ related, one a $\mathrm{SiO}_{2}$ substrate related signal and one a carbon bonded oxygen component. The $\mathrm{O} 1 \mathrm{~s}$ component peak at $531 \mathrm{eV}$ is attributed to the lattice oxygen in $\mathrm{ZnO}$, in agreement with the literature ${ }^{31}$, while the hydroxide peak $(\mathrm{ZnOH})$ is shifted by $1.5 \mathrm{eV}^{32,33}$ to higher binding energy. Neither of these peaks, nor their relative intensities, are significantly changed as a result of the UV radiation treatment even though a small increase in the intensity of the $\mathrm{OH}$ related component peak has been reported by other groups ${ }^{34-36}$ after UV irradiation, attributed to the desorption of $\mathrm{O} / \mathrm{O}_{2}$ species from the $\mathrm{ZnO}$ surface, which subsequently react with residual water vapour to form $\mathrm{OH}$ groups on the surface.

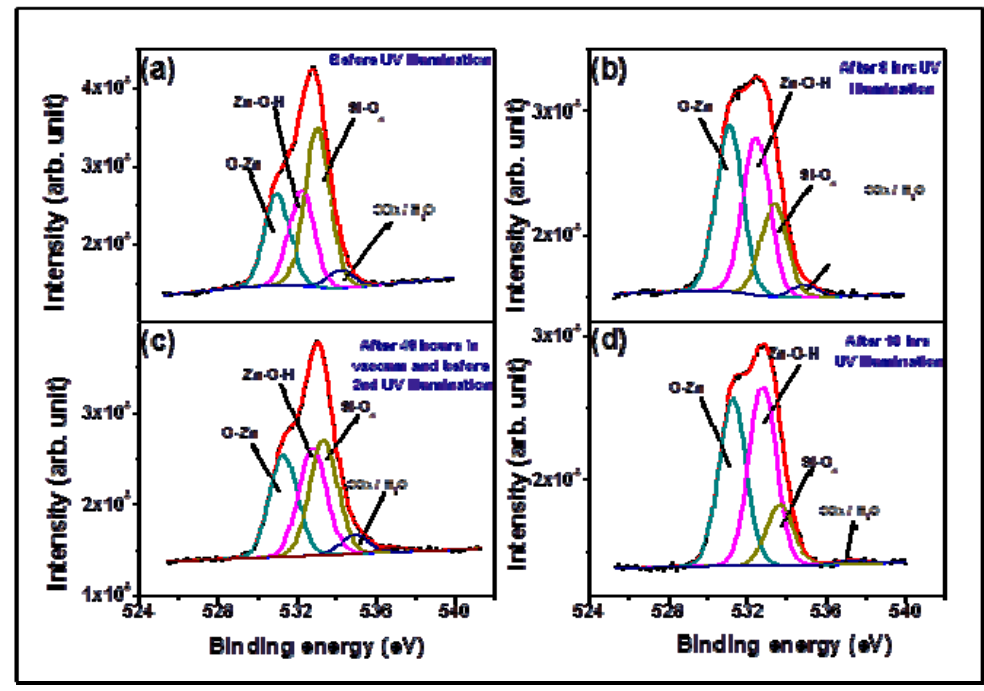

Figure 4: (Color online) Peak fitted O1s spectra at different stages of the illumination experiment. (a) before illumination, (b) after 6 hours illumination, (c) after leaving sample in vacuum for 48 hours before second illumination and (d) after 10 hours illumination.

The signal at $\sim 533.3 \mathrm{eV}$ in the $\mathrm{O} 1 \mathrm{~s}$ region is attributed to $\mathrm{O}$ bonded as $\mathrm{SiO},{ }^{29,30}$ due to the $\mathrm{SiO}_{2}$ native oxide on the substrate regions not covered by $\mathrm{Au}$ and $\mathrm{ZnO}$. This peak shows a substantial variation in intensity but this correlates with intensity changes seen in the Si2p region and is probably due to slight differences in the amount of the Si substrate illuminated from measurement to measurement. While this substrate derived component peak complicates the analysis and makes it difficult to compare the exact percentage change of the surface-adsorbed $O$ from peak fitting, it doesn't detract from the main observation that little change is observed in the $\mathrm{ZnOH}$ related feature as a function of UV irradiation. 


\subsection{Correlation of SX peak intensity to nanostructure morphology and crystal quality}

Figures 5(a) to 5(d) show SEM and PL spectra of ZnO nanorod/nanowall and nanorod samples grown at $900^{\circ} \mathrm{C}$ and $950^{\circ} \mathrm{C}$, respectively. The data from these two samples, and similar data from many other samples grown at $900^{\circ} \mathrm{C}$ and $950^{\circ} \mathrm{C}$ in our laboratory, ${ }^{7}$ have helped us form a general conclusion that an intense and broad SX peak is always observed for samples grown at $900{ }^{\circ} \mathrm{C}$ where nanorod/nanowall morphology is always observed and SX intensity is quite low for samples grown at $950{ }^{\circ} \mathrm{C}$ where nanorod only morphology (i.e. mostly nanorods, occasionally with some short walls at the base of nanorods) is observed.

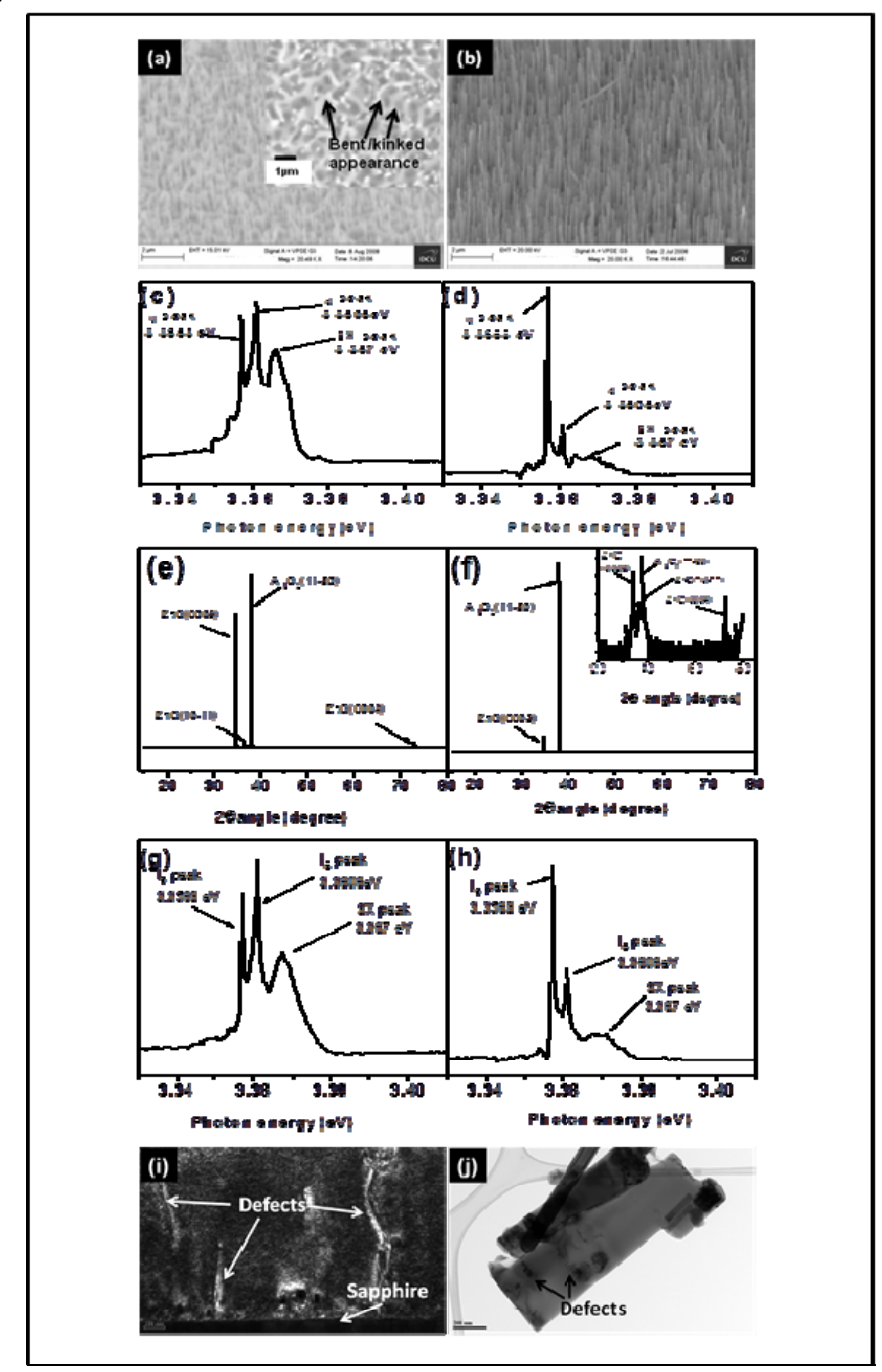

Figure 5: SEM images (a) \& (b) and PL spectra at $18 \mathrm{~K}$ (c) \& (d) of ZnO nanorod/nanowall sample grown at $900^{\circ} \mathrm{C} \mathrm{((a)} \mathrm{\&} \mathrm{(c))} \mathrm{and} \mathrm{ZnO}$ nanorod sample grown at $950^{\circ} \mathrm{C} \mathrm{((b)} \mathrm{\&} \mathrm{(d)).} \mathrm{Inset} \mathrm{of} \mathrm{(a)} \mathrm{shows}$ plan view SEM image of $\mathrm{ZnO}$ nanorod/nanowall sample shown in (a). XRD $2 \theta-\omega$ (e) \& (f) and PL data $(\mathrm{g}) \&(\mathrm{~h})$ of sample grown at $900^{\circ} \mathrm{C}((\mathrm{e}) \&(\mathrm{~g}))$ and sample grown at $950^{\circ} \mathrm{C}((\mathrm{f}) \&(\mathrm{~h}))$. Inset of (f) shows XRD $2 \theta-\omega$ data of $950^{\circ} \mathrm{C}$ grown sample on log scale. TEM images (i) and (j) of $\mathrm{ZnO}$ nanorod/nanowall samples grown at $900^{\circ} \mathrm{C}$. (PL intensity scales are linear). 
In figure 5 (e) and 5 (f), we show the $2 \theta-\omega$ scan of a typical $\mathrm{ZnO}$ nanorod/nanowall sample grown at $900^{\circ} \mathrm{C}$, and of a typical $\mathrm{ZnO}$ nanorod sample grown at $950^{\circ} \mathrm{C}$. We have studied a range of samples and have found a correlation between the SX PL peak and (10-11) XRD peak. For samples grown at $900{ }^{\circ} \mathrm{C}$ with intense SX peaks the (10-11) XRD peak is clearly visible on a linear intensity scale and for samples grown at $950{ }^{\circ} \mathrm{C}$ where the intensity of the SX peak is small the (10-11) XRD peak also being weak and only visible on a log scale. TEM studies have also been undertaken on both nanorod/nanowall and nanorod morphology samples. In figure 5 (i) and (j) TEM images of nanorod/nanowall samples grown at $900^{\circ} \mathrm{C}$ only are shown, as these show the nanowall structures with clear evidence of extended planar structural defects, such as grain boundaries, where nanowall structures have coalesced with nanorods during growth. By contrast, most of the actual nanorods in both $900^{\circ} \mathrm{C}$ and $950^{\circ} \mathrm{C}$ grown samples are found to be free of such extended structural defects (data not shown).

\section{Discussion}

The behaviour of the SX peak after electrical voltage application, presented in section 3.1, provides strong evidence for the possibility that SX emission is due to crystal defects confined to the adsorbed surface species which can bind excitons in their vicinity, in our view. The absence of a bias polarity or substrate carrier type dependence (and the absence of an effect for voltages less than $50 \mathrm{~V}$ ) in the voltage application results points to localized heating effects due to the electric current, which may affect both the adsorbed species on nanostructures in the vicinity of the contact and also the silver paste used for contacting (which can release moisture or other chemical species). The consistent recovery seen indicates however those heating effects do not lead to permanent effects due to sample annealing. Furthermore, it is also clear that voltage application has different effects in different atmospheres. The absence of any change in the inert He atmosphere suggests that the effect of the ambient may be to alter the density of available sites for adsorption of species (e.g. released by the heating effect of the current). Voltage application in vacuum may then lead to an uncompensated loss of a variety of weakly physisorbed species via desorption (uncompensated due to the low surrounding pressure) which then provides suitable accommodation sites for subsequent adsorption of the species responsible for the SX emission. By contrast, voltage application in air may lead to adsorption of species from the ambient, blocking suitable sites and perhaps even displacing some of the adsorbed species responsible for the SX emission. The consistent variations and recovery at room temperature or below of the SX PL in these experiments (and on timescales where significant contributions from defect drift and diffusion in the sub-surface region are not possible) and with differing, but consistent and reproducible, behaviour in different gas atmospheres provide strong evidence for the involvement of surface adsorbates in the SX PL emission as opposed to sub-surface crystal defects.

The two surface treatments of (a) plasma treatments and (b) UV illumination in high vacuum have both previously been shown to remove surface adsorbed oxygen species such as $\mathrm{O}_{2}^{-}, \mathrm{O}^{-}$and $\mathrm{O}^{2-}$ (and indeed photolysis of the $\mathrm{ZnO}$ surface also occurs when UV light of energy greater than the bandgap energy is incident on $\mathrm{ZnO}$ samples in high vacuum). ${ }^{18,20,37-42}$ However, as reported in sections 3.2 and 3.3 above, the absence of any significant or consistent changes in the SX peak either following both $\mathrm{O}$ and Ar plasma treatments or after illuminating with UV light in high vacuum lead us to conclude that the SX is not due to adsorbed $\mathrm{O} / \mathrm{O}_{2}$ on the nanostructure surfaces. 
The XPS data reported in section 3.4 clearly show that $\mathrm{OH}$ groups on the nanostructure surfaces are observed as strong and robust contributors to the spectra and are not removed by UV illumination in vacuum. Furthermore, XPS studies after in-situ annealing of this nanostructure sample up to $200^{\circ} \mathrm{C}$ (performed after the series of UV exposures and related XPS measurements reported above) show no variation in the $\mathrm{ZnO}$ and $\mathrm{OH}$ signals (data not shown). This behaviour shows how robust the $\mathrm{OH}$ contribution is and it is consistent with a broader range of our studies on other $\mathrm{ZnO}$ nanorod, thin film and single crystal samples which have been annealed up to $1000^{\circ} \mathrm{C}$ and exposed to cracked hydrogen at elevated temperatures $\left(400^{\circ} \mathrm{C}\right)$. Furthermore the sample shown in figure 4 , which exhibited intense SX PL, showed a stronger relative contribution from the OH XPS signal than other nanorod and planar samples. Also high temperature quenching of the PL of $\mathrm{ZnO}$ nanostructures which show strong SX emission at lower temperatures may be related to the presence of adsorbed $\mathrm{OH}$ species, which is in agreement with ref 48 where $\mathrm{OH}$ groups present on the surface of $\mathrm{ZnO}$ quantum dots are responsible for the quenching of bandedge excitonic emission. Thus, based on the XPS data reported in section 3.4 and figure 4, we find strong evidence for a relationship between the SX peak and the ubiquitous and robust $\mathrm{OH}$-related adsorbed surface species.

Finally, according to some reports in literature, SX peak has been attributed to a surface related defect with intensity determined solely or mainly by the surface to volume ratio., ${ }^{3,6,10,15}$ However, a number of reports of low temperature PL from $\mathrm{ZnO}$ nanostructures with varying aspect ratios show no consistent correlation from one report to another ${ }^{22-24}$ and within the same report ${ }^{2,15}$ between the SX peak relative intensity and the nanostructure aspect ratio. Based on the entirety of these reported data we question whether the SX peak's relative intensity is solely or mainly determined only by the nanostructure surface-to-volume ratio. From our studies reported in section 3.5 above, based on our observations of many samples grown at both $900^{\circ} \mathrm{C}$ and $950^{\circ} \mathrm{C}$, we conclude that the relative intensity of the SX peak is not solely dependent on the surface-to-volume ratio of the $\mathrm{ZnO}$ material. In addition, the XRD data indicate a different alignment of a fraction of the $\mathrm{ZnO}$ nanostructured deposit for samples with nanorod/nanowall morphologies which correlates with the SX peak emission intensity. Based on the relative intensities of the XRD peaks this fraction is likely to be small in terms of overall volume, but it may slightly affect the alignment of subsequent nucleation and growth of primarily (0001)-textured material leading to different regions of deposit with slightly differing crystallite orientations. This crystalline misalignment will lead to planar defects such as grain boundaries at the regions of coalescence of the differently aligned regions as growth proceeds and will influence, we believe, especially the coalescence of nanowall structures with nanorod bases. Planar defects of this type are in fact seen in the wall structures using TEM, as in figure 5 (i) and (j).

Based on these data we concludefirstly, the nanowall morphology displays a significantly bent/kinked appearance in plan view (shown in the inset of figure 5(a)) and these kink sites may offer a high density of attractive adsorption sites for $\mathrm{OH}$ species. Secondly, the presence of planar defects referred to above may also offer a high density of attractive adsorption sites for $\mathrm{OH}$ species at the regions where such defects intersect the nanostructure surfaces. Furthermore, adsorption may also occur at crystallite surfaces during nanostructure growth but prior to nanorod/nanowall coalescence leading to trapped $\mathrm{OH}$ species at such planar defects. The variety of slightly differing surface adsorption sites may also readily explain the generally observed large width $(\sim 5 \mathrm{meV})$ of the SX PL emission peak. There is evidence in support of this in the literature, including reports of low 
temperature $\mathrm{PL}$ from $\mathrm{ZnO}$ nanostructures with varying aspect ratios, which show no consistent correlation from one report to another between the SX peak relative intensity and the nanostructure aspect ratio (as well as variations with morphology variations seen within individual reports) see e.g. ref $15,22-24$.

\section{Conclusions}

The microscopic origins of the SX peak have been studied by low temperature PL in combination with various surface treatment methods, in addition to XRD, SEM and TEM. Voltage application in high vacuum, air and He gas show significant, consistent and recoverable changes in the peak intensity in the different gas atmospheres, providing clear evidence that the defects responsible are adsorbed surface species. High vacuum UV illumination and plasma treatments show no consistent or reproducible changes in the SX emission intensity and we conclude that $\mathrm{O} / \mathrm{O}_{2}$ is not the species responsible for the SX peak in $\mathrm{ZnO}$ nanostructure PL. XPS data show it is very difficult to remove the ubiquitous adsorbed $\mathrm{OH}$ species and that such species are strong candidates as the origin of the SX emission. XRD, SEM and TEM data show that the nanostructure morphology affects SX emission in a way not solely attributable to the surface to volume ratio.

Our final conclusion is that the SX peak is due to an exciton bound at the ZnO surface modified by an adsorbate, most likely an OH-related species and that the SX signal is strongly affected by the detailed nanostructure morphology. This work contributes to the understanding of the SX emission in $\mathrm{ZnO}$ nanostructures, which is an important issue in terms of device applications given the association of this emission with PL quenching at higher temperatures.

\section{Acknowledgements}

$\mathrm{MB}, \mathrm{EMcG}, \mathrm{GH}, \mathrm{KK}$ and $\mathrm{MOH}$ acknowledge financial support from an SFI-RFP grant (06/RFP/PHY052). HKK and YSJ acknowledge financial support from NSF grants (DMR 0216785 and ECCS 0403865). We are pleased to acknowledge the assistance of Dr. Ram Prasad Gandhiraman who grew $500 \mathrm{~nm} \mathrm{SiO}_{2}$ layers on Si substrates via PE-CVD.

\section{References}

[1] Meyer, B. K., Alves, H., Hofmann, D. M., Kriegseis, W., Forster, D., Bertram, F., Christen, J., Hoffmann, A., Straßburg, M., Dworzak, M., Haboeck, U., Rodina, A. V., "Bound exciton and donor-acceptor pair recombinations in ZnO," Phys. Status Solidi (b), 241(2), 231-260 (2004).

[2] Wischmeier, L., Voss, T., Ruckmann, I., Gutowski, J., Mofor, A. C., Bakin, A., and Waag, A., "Dynamics of surface-excitonic emission in ZnO nanowires," Phys. Rev. B 74(19), 195333-9 (2006).

[3] Stichtenoth, D., Ronning, C., Niermann, T., Wischmeier, L., Voss, T., Chien, C.-J., Chang, P.-C., and Lu, J. G., "Optical size effects in ultrathin ZnO nanowires," Nanotechnology, 18, 435701 (2007). 
[4] Brandt, O., Pfüller, C., Chèze, C., Geelhaar, L., and Riechert, H., "Sub-meV linewidth of excitonic luminescence in single GaN nanowires: Direct evidence for surface excitons," Phys. Rev. B, 81(4), 045302 (2010).

[5] Richters, J.-P., Voss, T., Wischmeier, L., Ruckmann, I., and Gutowski, J., "Influence of polymer coating on the low-temperature photoluminescence properties of $\mathrm{ZnO}$ nanowires," Appl. Phys. Lett., 92(1), 011103-3 (2008).

[6] Yang, Y., Tay, B. K., Sun, X. W., Sze, J. Y., Han, Z. J., Wang, J. X., Zhang, X. H., Li, Y. B., and Zhang, S., "Quenching of surface-exciton emission from $\mathrm{ZnO}$ nanocombs by plasma immersion ion implantation," Appl. Phys. Lett., 91(7), 071921-3 (2007).

[7] Grabowska, J., Meaney, A., Nanda, K. K., Mosnier, J. P., Henry, M. O., Duclere, J. R., and McGlynn, E., "Surface excitonic emission and quenching effects in $\mathrm{ZnO}$ nanowire/nanowall systems: Limiting effects on device potential," Phys. Rev. B 71(11), 115439-7 (2005).

[8] Travnikov, V. V., Freiberg, A., and Savikhin, S. F., "Surface excitons in $\mathrm{ZnO}$ crystals," J. Lumin., 47(3), 107-112 (1990).

[9] Richters, J. P., Voss, T., Kim, D. S., Scholz, R., and Zacharias, M., "Enhanced surfaceexcitonic emission in ZnO/A12O3 core\&ndash;shell nanowires," Nanotechnology, 19(30), 305202 (2008).

[10] Voss, T., and Wischmeier, L., "Recombination Dynamics of Surface-Related Excitonic States in Single ZnO Nanowires," J. of Nanosci. and Nanotechnol., 8, 228-232 (2008).

[11] Tenne, R., Nabutovsky, V. M., Lifshitz, E., and Francis, A. F., "Unusual photoluminescence of porous CdS (CdSE) crystals," Solid State Commun., 82(9), 651-654 (1992).

[12] Travnikov, V. V., "Surface radiative recombinationin CdS crystals," J. Crys. Growth, 101(14), 579-583 (1990).

[13] Travnikov, V. V., "Surface and volume emission of excitons in theluminescence spectra of CdS crystals " JETP Letters, 40, 1060 (1984).

[14] Lapeyre, G. J., and Anderson, J., "Evidence for a Surface-State Exciton on GaAs(110)," Phys. Rev. Lett., 35(2), 117 (1975).

[15] Wischmeier, L., Voss, T., Börner, S., and Schade, W., "Comparison of the optical properties of as-grown ensembles and single ZnO nanowires," Appl. Phys. A, 84(1), 111-116 (2006).

[16] Voss, T., Richters, J.-P., and Dev, A., "Surface effects and nonlinear optical properties of ZnO nanowires," Phys. Status Solidi (b), 247(10), 2476-2487 (2010).

[17] Fan, H. J., Barnard, A. S., and Zacharias, M., "ZnO nanowires and nanobelts: Shape selection and thermodynamic modeling," Appl. Phys. Lett., 90(14), 143116-3 (2007).

[18] Lagowski, J., Sproles, J. E. S., and Gatos, H. C., "Quantitative study of the charge transfer in chemisorption; oxygen chemisorption on ZnO," J. Appl. Phys., 48(8), 3566-3575 (1977).

[19] Bickley, D. R. I., Heiland, P. G., Hirschwald, D. W., Thull, D. E., Steinbach, P. F., Harborth, M. R., Bauer, D. W., Hausmann, P. A., Tench, D. A. J., Cunningham, P. J., Roberts, P. M. W., Grimley, D. T. B., Menzel, P. D., and Boehm, P. H. P., "General discussion on UV effect on ZnO" Faraday Discuss. Chem. Soc. 58, 175-184 (1974).

[20] Steinbach, F., and Harborth, R., "Oxidation of CO and desorption of oxygen by ultra-violet irradiation of $\mathrm{ZnO}$ single crystals under ultra-high vacuum conditions" Faraday Discuss. Chem. Soc. 58, 143-150 (1974).

[21] Zhang, Y. Z., He, H. P., Jin, Y. Z., Zhao, B. H., Ye, Z. Z., and Tang, H. P., "Reduced bound exciton and surface exciton emissions in Al-doped $\mathrm{ZnO}$ nanorods exposed to ambient air," J. Appl. Phys., 104(10), 103529-5 (2008). 
[22] Zhou, H., Fallert, J., Sartor, J., Dietz, R. J. B., Klingshirn, C., Kalt, H., Weissenberger, D., Gerthsen, D., Zeng, H., and Cai, W., “Ordered n-type ZnO nanorod arrays," Appl. Phys. Lett., 92(13), 132112-3 (2008).

[23] Li, Y., Feneberg, M., Reiser, A., Schirra, M., Enchelmaier, R., Ladenburger, A., Langlois, A., Sauer, R., Thonke, K., Cai, J., and Rauscher, H., "Au-catalyzed growth processes and luminescence properties of ZnO nanopillars on Si,” J. Appl. Phys., 99(5), 054307-9 (2006).

[24] Reshchikov, M. A., Behrends, A., Bakin, A., and Waag, A., "Photoluminescence from $\mathrm{ZnO}$ nanowires," J. Vac. Sci. Technol. B, 27(3), 1688 (2009).

[25] Grabowska, J., Nanda, K. K., McGlynn, E., Mosnier, J. P., and Henry, M. O., "Studying the growth conditions, the alignment and structure of $\mathrm{ZnO}$ nanorods," Surf. Coat. Technol., 200(1-4), 1093-1096 (2005).

[26] Biswas, M., McGlynn, E., Henry, M. O., McCann, M., and Rafferty, A., "Carbothermal reduction vapor phase transport growth of $\mathrm{ZnO}$ nanostructures: Effects of various carbon sources," J. Appl. Phys., 105(9), 094306-6 (2009).

[27] Moulder, J. F., Stickle, W. F., E.Sobol, P., and Bomben, K. D., [Handbook of X-Ray Photoelectron Spectroscopy] 1st ed., ed. J. Chastain, Perkin-Elmer Corporation: Eden Prairie, Mn., (1992).

[28] Wöll, C., "The chemistry and physics of zinc oxide surfaces," Prog. Surf. Sci., 82(2-3), 55120 (2007).

[29] http://srdata.nist.gov/xps/.

[30] Lupan, O., Chow, L., Chai, G., Roldan, B., Naitabdi, A., Schulte, A., and Heinrich, H., "Nanofabrication and characterization of $\mathrm{ZnO}$ nanorod arrays and branched microrods by aqueous solution route and rapid thermal processing," Mater. Sci. Eng. B, 145(1-3), 57-66 (2007).

[31] Wagner, C. D., and Muilenberg, G. E., [Handbook of x-ray photoelectron spectroscopy] Physical Electronics Division, Perkin-Elmer Corp., (1979).

[32] Elizabeth Pugel, D., Vispute, R. D., Hullavarad, S. S., Venkatesan, T., and Varughese, B., "Compositional origin of surface roughness variations in air-annealed $\mathrm{ZnO}$ single crystals," Appl. Surf. Sci., 254(8), 2220-2223 (2008).

[33] Barr, T. L., "An ESCA study of the termination of the passivation of elemental metals," The J. Phys. Chem., 82(16), 1801-1810 (1978).

[34] Sun, R.-D., Nakajima, A., Fujishima, A., Watanabe, T., and Hashimoto, K., "Photoinduced Surface Wettability Conversion of ZnO and TiO2 Thin Films," J. Phys. Chem. B, 105(10), 1984-1990 (2001).

[35] Asakuma, N., Fukui, T., Toki, M., Awazu, K., and Imai, H., "Photoinduced hydroxylation at ZnO surface," Thin Solid Films, 445(2), 284-287 (2003).

[36] Asakuma, N., Hirashima, H., Fukui, T., Toki, M., Awazu, K., and Imai, H., "Photoreduction of amorphous and crystalline ZnO films,” Jpn. J. Appl. Phys., 41(6A), 3909-3915 (2002).

[37] Zhang, D. H., "Fast photoresponse and the related change of crystallite barriers for ZnO films deposited by RF sputtering," J. Phys. D: Appl. Phys., 28(6), 1273 (1995).

[38] Cunningham, J., Finn, E., and Samman, N., "Photo-assisted surface reactions studied by dynamic mass spectrometry," Faraday Discuss. Chem. Soc. 58, 160-174 (1974).

[39] Jin, C., Tiwari, A., and Narayan, R. J., "Ultraviolet-illumination-enhanced photoluminescence effect in zinc oxide thin films," J. Appl. Phys., 98(8), 083707-7 (2005). 
[40] Keem, K., Kim, H., Kim, G.-T., Lee, J. S., Min, B., Cho, K., Sung, M.-Y., and Kim, S., "Photocurrent in $\mathrm{ZnO}$ nanowires grown from $\mathrm{Au}$ electrodes," Appl. Phys. Lett., 84(22), 4376-4378 (2004).

[41] Brundle, C. R., Evans, S., Roberts, M. W., Briggs, D., Fuggle, J. C., Menzel, D., Smith, F. R., Thomas, J. M., Parry, D. E., Bradshaw, A. M., Biloen, P., Plummer, E. W., Grimley, T. B., Lloyd, D. R., Joyner, R. W., Kuppers, J., and Killesreiter, H., "General discussion," Faraday Discuss. Chem. Soc., 58, 125-142 (1974).

[42] Liu, M., and Kim, H. K., "Ultraviolet detection with ultrathin $\mathrm{ZnO}$ epitaxial films treated with oxygen plasma," Appl. Phys. Lett., 84(2), 173-175 (2004). 